\title{
Physiological and Relational Predictors of Mother-Infant Behavioral Coordination
}

\author{
Sara F. Waters ${ }^{1} \cdot$ Wendy Berry Mendes ${ }^{2}$
}

Received: 13 November 2015 / Revised: 4 April 2016 / Accepted: 6 April 2016 /

Published online: 20 April 2016

C) Springer International Publishing 2016

\begin{abstract}
Coordinated social behavior and positive affect shared between parent and child in early life provide a foundation for healthy social and emotional development. We examined physiological (cardiac vagal responses) and relational (attachment security) predictors of dyadic behavioral coordination in a sample of 13-month-old infants and their mothers $(N=64)$. We tested whether cardiac vagal responses moderated the association between attachment security and behavioral coordination. The main effect of attachment on coordination was moderated by infant cardiac vagal tone (i.e., respiratory sinus arrhythmia [RSA] during rest). Securely attached infants with lower cardiac vagal tone were more behaviorally coordinated with their mothers; there was no association between attachment and coordination for infants with high cardiac vagal tone. Infants with greater increases in cardiac vagal reactivity (i.e., RSA during social engagement) exhibited greater behavioral coordination with their mothers regardless of attachment status. There were no effects for maternal cardiac vagal responses. These results illustrate how individual differences in physiological responses inform healthy early social-emotional functioning.
\end{abstract}

Keywords Cardiac vagal tone $\cdot$ Cardiac vagal reactivity $\cdot$ RSA $\cdot$ Attachment $\cdot$ Dyad

The building blocks for lifelong social-emotional functioning are established by the quality of early parent-child interactions. Regular experiences of shared positive affect

Sara F. Waters

sara.f.waters@wsu.edu

Wendy Berry Mendes

wendy.mendes@ucsf.edu

1 Department of Human Development, Washington State University Vancouver, 14204 NE Salmon Creek Avenue, Vancouver, WA 98686, USA

2 Department of Psychiatry, University of California San Francisco, 410 Parnassus Avenue, San Francisco, CA 94143-0984, USA 
and coordinated social behavior between parent and child in the first few years of life positively predict children's social-emotional skills and are negatively associated with behavior problems (Deater-Deckard and Petrill 2004; Lindsey et al. 2009; Lindsey et al. 1997). For instance, children who were more socially coordinated with their mothers during interactions at 9 months of age had greater emotional self-regulation at 2 years of age (Feldman et al. 1999). This is important because children who are well-regulated and have positive social interactions with others demonstrate greater psychological adjustment and academic achievement (Gaziano et al. 2007; Spinrad et al. 2006). In another study, positivity of mother-child interactions at 14 months of age predicted children's tendency to imitate the mother and the child's level of distress to breaking an object 8 months later (Kochanska et al. 1999). Imitation during interaction and negative emotionality over (perceived) responsibility for a social transgression are constituents of a prosocial orientation (Cole et al. 1992; Frankel and Bates 1990). As positive interactive coordination between mother and infant fosters positive social-emotional development, it is important to understand correlates of this coordination.

\section{Attachment Security}

One such correlate may be the attachment bond that forms between the primary caregiver and the infant over the first year of life. The quality of this bond, whether it is secure or insecure, has significant implications for children's social-emotional development (Bowlby 1982). Attachment theorists posit that when a caregiver is consistently and appropriately responsive to the infant's emotional cues, the infant learns that the caregiver can be depended on to care for and soothe him, forming a secure attachment (Ainsworth 1979; Thompson 1994). Securely attached children demonstrate greater willingness to engage with new features of the environment (Grossmann et al. 1999) and attachment security has been linked to a variety of positive social-emotional behaviors (Bohlin et al. 2000; Boldt et al. 2014). Not all studies find this direct relationship, however, and contextual risk is an important moderator (Belsky and Pasco Fearon 2002). Few studies to date have examined moderation by individual differences in physiological responses to explain discrepancies in associations between attachment security and social-emotional outcomes.

\section{Cardiac Vagal Response}

The tenth cranial nerve, the vagus, is one mechanism by which the brain, specifically, can act on the heart's pacemaker and regulate heart rate and heart rate variability in response to changes in the environment. Vagal influence can be quantified in terms of heart rate variability or variability in the beat-to-beat patterns of the heart. There are several methods for measuring heart rate variability and respiratory sinus arrhythmia (RSA) is the most well established in the research literature. RSA is beat-to-beat variability within the frequency of heart rate periodicity associated with spontaneous breathing. Cardiac vagal tone refers to RSA at rest and cardiac vagal reactivity refers to the change in RSA from rest to a task or activity. The research examining correlates of cardiac vagal tone and reactivity in adults and young children has been mixed, 
but some important patterns emerge indicating RSA may be a "biomarker" of emotional functioning and risk for psychopathology (Beauchaine 2015; Beauchaine and Thayer 2015).

One body of research has focused on adult clinical samples, consistently finding that low cardiac vagal tone or resting RSA is associated with various psychiatric difficulties (Demaree and Everhart 2004; Friedman and Thayer 1998; Licht et al. 2008). Less work to date speaks to vagal tone in healthy individuals, but high cardiac vagal tone has been linked to dispositional positive emotion in healthy college students (Oveis et al. 2009). Studies of both adults and young children find that high cardiac vagal tone is associated with emotional reactivity. Whether this reactivity involves increased expression of positive or negative emotion depends on the context (Butler et al. 2006; Calkins and Fox 1992; Fox, 1989; Stifter et al. 1989). For instance, when faced with an emotionally challenging task, high vagal tone infants expressed more negative emotion than low vagal tone infants. When the context was social and affectively positive, like a game of peek-a-boo, high vagal tone infants expressed more positive emotion than low vagal tone infants. Thus, Beauchaine (2001) has argued that high cardiac vagal tone is a marker of physiological flexibility that supports adaptive engagement with the environment. To more fully understand the relationship between cardiac vagal tone and social-emotional behavior in infants and young children, we must consider parenting influences as well.

The results of extant research indicate several different ways in which cardiac vagal tone, socialization, and children's behavioral outcomes are associated. Children with higher cardiac vagal tone have been found to be more susceptible to parental influences - when attachment bonds are secure or parenting is good, children with higher cardiac vagal tone have better outcomes and when attachment bonds are insecure or parenting is poor, children with higher cardiac vagal tone have worse outcomes (Conradt et al. 2013; Eisenberg et al. 2012). Other studies have found the opposite relationship children with lower cardiac vagal tone, but not higher cardiac vagal tone, have poorer behavioral outcomes when parenting is problematic or less optimal (Dierckx et al. 2011; Hastings et al. 2008). More research like the current study is needed to shed light on these complex and sometimes contradictory associations.

Polyvagal theory (Porges 1995; 2007) argues that the activity of the vagal nerve on the heart, or cardiac vagal reactivity, is a biological indicator of self-regulation. Increased vagal influence as indexed by increases in RSA from rest to task supports a state of calm and activates a social engagement system throughout the body. Withdrawal of vagal influence indexed by decreases in RSA from rest to task, on the other hand, supports "fight or flight" behaviors presumably in response to a stressor or challenge in the environment. Some adult research supports this claim with a relaxation task increasing RSA (Houtveen et al. 2002) while in other work the results are more nuanced (Frazier et al. 2004). The literature on young children finds more consistently that increases in RSA are associated with positive social interactions (Gentzler et al. 2009; Ham and Tronick 2006; Oosterman et al. 2010) while challenging tasks produce decreases in RSA from rest (Blandon et al. 2008; Huffman et al. 1998).

There is less research focused on cardiac vagal reactivity than cardiac vagal tone, especially with regards to cardiac vagal reactivity as a moderator of associations between parenting and child outcomes. Holochwost and colleagues (2014) found that a lack of RSA decrease during parent-child interactions, when combined with intrusive 
parenting, fostered the development of a disorganized attachment. In an at-risk sample, children who had lower rates of violence exposure and greater decreases in RSA to challenging laboratory tasks exhibited better emotional adjustment (Cipriano et al. 2011). Based on a handful of studies, less cardiac vagal reactivity during a task may indicate a susceptibility to negative socialization influences. The current study contributes to our developing understanding of the possibility of cardiac vagal reactivity as a moderator of parent-child relationship factors on social-emotional behavior.

In the current study, we examined the influence of infant attachment security and infant and maternal cardiac vagal tone and reactivity on mother-infant behavioral coordination. Consistent with attachment theory, we hypothesized that more securely attached infants would be more behaviorally coordinated with mothers. Based on the evidence that high cardiac vagal tone supports environmental engagement, we hypothesized that mothers and infants with higher cardiac vagal tone would be more behaviorally coordinated. In line with the claim that cardiac vagal reactivity indexes emotional self-regulation, we hypothesized that mothers and infants with increases in RSA from test to a social engagement task would be more behaviorally coordinated as well. We tested interaction effects of cardiac vagal tone and attachment security and cardiac vagal reactivity and attachment security on behavioral coordination for both infants and mothers. Given the relatively scant and mixed findings, we did not have firm hypotheses for these moderation analyses.

\section{Method}

\section{Participants}

Sixty-four mothers $\left(M_{\text {age }}=32.94\right.$ years, $\left.S D=5.6\right)$ and their 12 - to 14 -month-old infants $\left(M_{\text {age }}=13.12\right.$ months, $S D=1.21 ; 48.5 \%$ female $)$ were recruited from the San Francisco Bay Area via on-site (e.g. parenting classes) and online (e.g. Craigslist, mothers' group blogs) advertisements. Participating families represented a range of ethnic and socioeconomic backgrounds. Fifty-eight percent of mothers were European American, 19\% were Asian American, 9\% were multi-ethnic, 7\% were Latina, and 7\% were African American or biracial. Twenty percent of mothers had a high school degree, $6 \%$ had an associate's degree, 44\% had a bachelor's degree, and 30\% had a master's or doctorate degree. Thirteen percent of participating families earned less than $\$ 25,000$ a year, $19 \%$ earned between $\$ 25,000$ and $\$ 50,000,26 \%$ earned between $\$ 50,000$ and $\$ 100,000$, $26 \%$ earned between $\$ 100,000$ and $\$ 150,000$, and $15 \%$ earned over $\$ 150,000$ a year. Nearly all mothers $(96 \%)$ were married or cohabitating with the infant's father. Mothers were compensated $\$ 75$ for participation with their infants and travel costs when appropriate.

\section{Procedure}

In the weeks before the laboratory visit, each mother was mailed a packet of study information including the Attachment Q-sort (AQS) with instructions for how to complete it. Upon arrival at the laboratory, the mother provided consent for herself and her infant. The infant was taken to a playroom by a familiar caregiver while an 
experimenter went through the AQS with the mother, answered any questions, and confirmed that all cards were sorted appropriately following a protocol adapted from Teti and McGourty (1996) and Laible and Thompson (1998). The experimenter then applied sensors (see below) to the mother and instructed her to sit quietly while a 5minute resting baseline of the mother's physiological responses was recorded. The infant was returned to the mother and sensors were applied. It can be challenging to obtain several minutes of artifact-free physiological data on infants without engaging them in an activity like watching a video (i.e., Conradt and Ablow 2010). These tasks have attentional demands that may influence the infant's physiological responses (Richards 1987). Thus the experimenter instructed the mother to help her infant relax without the use of toys or games and left the dyad alone with some pleasant music for a 2-minute rest period. This approach is used for obtaining valid resting physiological responses from infants with minimal attentional demands (see Alkon et al. 2006).

Following mother baseline and infant rest period, the infant returned to the playroom while the mother was randomly assigned to complete a speech with negative $(n=27)$, positive $(n=23)$ or no $(n=23)$ evaluative feedback. The speech task was part of a separate research question that was not relevant to the focus of the current study and the speech task is not presented here in further detail (see Waters et al. 2014). Speech condition was entered as a control variable in all analyses.

The infant rejoined the mother following the speech task and physiological responses were obtained continuously during the subsequent interaction episodes, which consisted of a 2-minute reunion, 2-minute second rest episode, and two 2-minute social interviews with an experimenter. In the reunion episode, mother and infant were left alone to reconnect. The second rest episode followed the same format as the first rest in which pleasant background music was played while the mother was instructed to help her infant relax. In the social interviews, each of two female experimenters engaged mother and infant in an innocuous conversation and a social bid (i.e. offered the infant a toy).

The final episode of the laboratory visit was a 3-minute toy-offer episode in which the two female experimenters from the social interviews entered the room together and engaged the dyad in a series of social bids (i.e. offered the infant several new toys). This toy-offer episode was videotaped for later behavioral coding. Upon completion of the study, the sensors were detached, the mother was debriefed, and payment was given.

\section{Measures}

Respiratory Sinus Arrhythmia (RSA) Electrocardiography was collected on mothers and infants from two spot sensors on the chest using the Biopac MP150 Data Acquisition System (Biopac Systems, Inc., www.biopac.com). This system allows multiple channels of physiological data to be acquired on multiple individuals simultaneously. Thus mother and infant electrocardiographic data were time-synced at recording. Trained research assistants who were blind to study hypotheses and maternal speech condition visually inspected waveforms for artifacts using Mindware software (Heart Rate Variability Analysis Software 2.6, Mindware Technologies, Ltd., http://www.mindwaretech.com/) before aggregating the data in 30-second segments. Consistent with the literature, a high frequency band from 0.12 to 0.40 was used to derive maternal RSA data and a high frequency band from 0.24 to 1.04 was used to 
derive infant RSA data (Bar-Haim et al. 2000). Cardiac vagal tone was calculated by averaging the RSA values from the last minute of the baseline and rest period for mothers and infants respectively, which is presumably when they were the most acclimated to the sensors. Cardiac vagal reactivity was calculated by subtracting the cardiac vagal tone value from the RSA value during each of the 2 min of the interview. This produced a measure of each partner's cardiac vagal responses while interacting with a different social partner than the other dyad member. The two 1-minute reactivity scores were then averaged to create a single measure of cardiac vagal reactivity to the social interview episode.

Infant Attachment Security The Attachment Q-sort version 3.0 (AQS; Waters and Deane 1985) measures the security of the infant-mother attachment bond, and is a commonly used measure of attachment in infants and young children that produces a single continuous measure of attachment security. Each of the 90 cards in the Q-sort contains a descriptive statement, 30 of which are "filler" statements that are unrelated to attachment security. Each card has been assigned a value reflecting the score of a prototypically "most secure" child. Mothers sorted the cards into nine groups based on how accurately they described their child. Security scores on a continuous scale from -1.0 to 1.0 were then calculated by correlating the observed child's scores on the Q-sort items with the security criterion sort containing values for the prototypically secure child. A security score over .30 is indicative of a secure attachment (Waters and Deane 1985). Security scores were examined for outliers greater than $3 S D$ from the sample mean and one dyad was excluded according to this criterion.

Behavioral Coordination Mother-infant behavioral coordination during the toy-offer episode was coded by trained observers using a 5-point scale anchored at 0 (dyad was not coordinated) to 4 (dyad exhibited consistent coordination throughout). Indicators of behavioral coordination involved instances of verbal and nonverbal reciprocated communication including eye contact, smiles, vocalizations, and interactive toy play (e.g. mother hands toy to infant and infant accepts it). Two female research assistants, unaware of any study manipulations or participant information, trained with the first author and achieved reliability with each other on a random $40 \%$ of the sample $(I C C=.76)$ before one of them coded the remaining videotapes singly.

\section{Data Analysis}

Analyses were conducted using SPSS 22.0. Descriptive statistics were calculated for all study variables. Significance levels were set at $p<.05$. Pearson correlation coefficients were calculated to measure associations among continuous variables while Spearman's rho was used for nominal variables (i.e. mother speech condition). We conducted separate hierarchical linear regressions predicting behavioral coordination from mother cardiac vagal responses and infant cardiac vagal responses. Each regression included attachment security, cardiac vagal tone, cardiac vagal reactivity, and the two attachment security by cardiac vagal response interaction terms. Maternal speech condition was included as control variable in the models. We unpacked significant interaction terms using simple slopes (Aiken and West 1991) and comparing the effect of attachment 
security on behavioral coordination at high (1 SD above the mean) and low (1 SD below the mean) levels of vagal response.

\section{Results}

Preliminary analyses confirmed that the cardiac vagal variables were normally distributed while attachment security was skewed. Thus Spearman's rho was used for bivariate analyses with attachment security. Descriptive statistics and bivariate associations among study variables are presented in Table 1. On average, infants met the threshold for secure attachment but there was considerable range in attachment scores. Infants, on average, exhibited increases in RSA from rest period to the interview episode as represented by a positive reactivity score. As one would expect, infant cardiac vagal tone was highly correlated with infant cardiac vagal reactivity. The same association was found between maternal cardiac vagal tone and reactivity.

\section{Infant Cardiac Vagal Responses Model}

The full hierarchical linear regression model predicting behavioral coordination from attachment security and infant cardiac vagal responses was significant, $F(6,46)=4.62, p=.001$, $R^{2}=.37, R_{a d j}{ }^{2}=.29$. As shown in Table 2, there were two notable significant main effects. The first was that more securely attached infants displayed greater behavioral coordination with mothers, as objectively observed. The second main effect was that infants who exhibited greater increases in RSA from rest period to the social interview with a novel adult displayed greater behavioral coordination with mothers as well. There was no main effect of cardiac vagal tone on behavioral coordination. Instead, vagal tone moderated the relationship between infant attachment security and behavioral coordination (Fig. 1). For infants with lower cardiac vagal tone, greater attachment security predicted greater behavioral coordination, $t(49)=3.73 p<.001$. For infants with higher cardiac vagal tone, however, there was no relationship between attachment security and behavioral coordination, $t(49)=-0.80, p=.43$. Another way to consider this association is that secure attachment buffered infants with lower levels of vagal tone whereas those with higher vagal tone showed strong behavioral coordination regardless of the level of attachment.

Table 1 Descriptive statistics and bivariate associations among study variables

\begin{tabular}{|c|c|c|c|c|c|c|c|c|}
\hline & Mean $(S D)$ & Range & 1 & 2 & 3 & 4 & 5 & 6 \\
\hline 1. Mother speech condition ${ }^{\mathrm{a}}$ & $\mathrm{N} / \mathrm{A}$ & N/A & & & & & & \\
\hline 2. Infant cardiac vagal tone & $3.96(0.98)$ & $1.87-6.59$ & .02 & & & & & \\
\hline 3. Infant cardiac vagal reactivity & $0.11(0.88)$ & $-2.20-1.87$ & -.13 & $-.56^{* *}$ & & & & \\
\hline 4. Maternal cardiac vagal tone & $6.45(1.13)$ & $4.14-9.12$ & -.06 & -.01 & .20 & & & \\
\hline 5. Maternal cardiac vagal reactivity & $.01(1.0)$ & $-3.0-2.25$ & -.17 & $.28^{*}$ & $-.35^{* *}$ & $-.50 * *$ & & \\
\hline 6. Attachment security ${ }^{\mathrm{a}}$ & $0.33(0.19)$ & $-0.20-0.72$ & .10 & .08 & -.05 & -.19 & .12 & \\
\hline 7. Behavioral coordination & $3.14(1.3)$ & $1-5$ & -.07 & -.18 & $.31 *$ & .11 & -.19 & $.22+$ \\
\hline
\end{tabular}

${ }^{\text {a }}$ Spearman's rho. $+p<.10 . * p<.05 ; * * p<.01$ 
Table 2 Predicting behavioral coordination from attachment security and infant cardiac vagal responses

\begin{tabular}{|c|c|c|c|c|c|c|}
\hline & \multirow[t]{2}{*}{$b$} & \multirow[t]{2}{*}{$S E$} & \multirow[t]{2}{*}{$B$} & \multirow[t]{2}{*}{$t$} & \multicolumn{2}{|c|}{$95 \% \mathrm{CI}$} \\
\hline & & & & & Lower & Upper \\
\hline Mother speech condition & -0.16 & 0.19 & -0.10 & -0.85 & -0.55 & 0.22 \\
\hline Infant cardiac vagal tone & 0.09 & 0.22 & 0.07 & 0.48 & -0.30 & 0.48 \\
\hline Infant cardiac vagal reactivity & 0.48 & 0.22 & 0.32 & $2.24 *$ & 0.05 & 0.92 \\
\hline Attachment security & 2.84 & 0.88 & 0.39 & $2.24 *$ & 1.08 & 4.61 \\
\hline Attachment $\mathrm{X}$ infant cardiac vagal tone & -5.51 & 1.29 & -0.64 & $-3.70 * *$ & -8.51 & -2.51 \\
\hline Attachment $\mathrm{X}$ infant cardiac vagal reactivity & -2.39 & 1.26 & -0.32 & -1.91 & -4.92 & 0.13 \\
\hline
\end{tabular}

$b=$ unstandardized; $\beta=$ standardized $* p<.05 ; * * p .01$

\section{Maternal Cardiac Vagal Responses Model}

The full model predicting behavioral coordination from attachment security and maternal cardiac vagal responses was marginally significant, $F(6,53)=1.43, p=.22$, $R^{2}=.14, R_{a d j}{ }^{2}=.04$. This model was driven by the significant positive predictor of infant attachment security described above, $p=.03$. Neither maternal cardiac vagal tone nor reactivity demonstrated significant main effect ( $p s=.83$ and .31 , respectively) nor did they moderate the influence of infant attachment security ( $p s=.35$ and .64 , respectively) on behavioral coordination. In contrast to infants' responses, maternal cardiac vagal responses did not contribute to the quality of behavioral coordination in the mother-infant toy play episode.

\section{Discussion}

We investigated attachment security and cardiac vagal responses as predictors of mother-infant behavioral coordination, an important antecedent of healthy socialemotional development. The findings partially support our hypotheses and align with tenets of attachment theory and polyvagal theory. Securely attached infants and infants with greater increases in cardiac vagal responses when interacting with a stranger

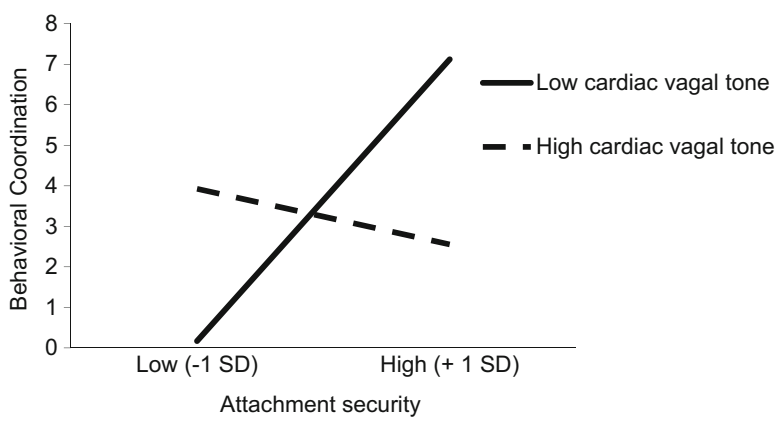

Fig. 1 Infant cardiac vagal tone moderates the influence of attachment security on mother-infant behavioral coordination 
displayed greater behavioral coordination with their mother. Additionally, infants' cardiac vagal tone moderated the association between attachment and coordination. Infants who had lower cardiac vagal tone displayed greater behavioral coordination with their mothers only when securely attached to them. Infants with lower cardiac vagal tone and an insecure attachment displayed low behavioral coordination with their mothers. We did not observe any associations between maternal cardiac vagal responses and behavioral coordination.

In this study, we unpacked the influence of cardiac vagal reactivity separately from cardiac vagal tone. Infants for whom RSA increased from rest to the social interview task (i.e. less cardiac vagal reactivity) demonstrated greater coordination with mothers in a subsequent interaction. This effect emerged over and above the predictive power of attachment security and cardiac vagal tone. Changes in RSA can be associated with various physical or psychological states. From the perspective of polyvagal theory, infants with greater increases in cardiac vagal reactivity may have maintained a socially engaged stance during an interaction with a novel female rather than a more physiologically activated one and this may have supported high quality interactions with mothers. In a recent synthesis of the RSA literature, high vagal reactivity to an emotional challenge was identified as a biomarker of poor emotion regulation (Beauchaine 2015). Our results align with this claim as infants with high vagal reactivity may not have had the regulatory abilities to needed to support a behaviorally coordinated interaction with mothers. We did not find that reactivity moderated the effect of attachment security, however. Together these two results indicate that cardiac vagal reactivity is a potent predictor of social-emotional behavior independent of relationship quality.

In contrast, infant cardiac vagal tone was not directly associated with behavioral coordination, but moderated the impact of attachment security on it. Infants with lower vagal tone needed to be securely attached to manifest positive interaction patterns, but infants with higher vagal tone exhibited moderate levels of behavioral coordination regardless of attachment security. Thus, higher infant vagal tone emerged as a protective factor against negative social-emotional behavior associated with an insecure attachment. Again, this finding aligns with Beauchaine's (2015) argument that low vagal tone is a biomarker of poor emotion regulation. Infants with low vagal tone, and thus perhaps poorer emotion regulation abilities, needed the history of a positive, securely attached relationship to engage in behavioral coordination with mothers. Consistent with our results, some developmental studies have found that low vagal tone signaled sensitivity to the quality of parenting (Hastings et al. 2008), while others have found moderation by high vagal tone (Conradt et al. 2013; Eisenberg et al. 2012). It is important to note that the studies finding high vagal tone as a moderator involved samples with large proportions of low-income families while our sample was relatively high in resources. Differences in the socioeconomic environments of the samples may help explain these discrepancies. These discrepancies may also reflect timing of assessments with associations of higher vagal tone with negative outcomes potentially indexing sensitivity to context and resulting in poorer outcomes in harsher environments, and associations of lower vagal tone with negative outcomes resulting from extended exposure.

Mothers are more socially and emotionally sophisticated than infants and our outcome measure of mother-infant coordination was fundamentally dyadic in nature. 
Thus it is noteworthy that neither of the maternal physiological responses was associated with behavioral coordination. These findings indicate that individual differences in maternal physiology may be less impactful on key features of the mother-infant interaction than infant physiology. Recent evidence suggests that an individual's physiological responses influence and are influenced by their partner's physiological responses as they unfold over time (Ferrer and Helm 2013). Perhaps maternal physiology is not associated with behavioral coordination directly, but through its influences on infant physiology.

There are some limitations to the current study to consider. The most commonly used measure of infant attachment security, the Strange Situation, was not feasible to employ within the study design. The single lab visit involved presenting mothers with a mildly stressful task and we felt that extending the protocol to include the Strange Situation, a mild to moderate stress task, risked overwhelming infants and making participation untenable. Mothers may be biased in their reporting on attachment with their infant so the AQS was designed with "filler" items meant to obscure the specific goal of the measure and reduce systematic bias. Mother-reported AQS allowed us to draw each of our measures from different sources, which strengthens what can be concluded from the findings. Attachment theory recognizes that an attachment bond emerges from the accumulation of mother-infant interactions over the first year and our attachment security score was based upon the totality of the mother's experiences with her infant during that year. Thus our model, in which we predicted an episode of socialemotional dyadic behavior from a history of relationship quality with cross-sectional data, was firmly grounded in theory. While mothers received varying forms of feedback in the speech task they completed before being reunited with their infants, there was no evidence of speech condition effects on mother or infant RSA data, infant attachment security or behavioral coordination.

In sum, the current investigation shed light on how relational and physiological characteristics are associated with mother-infant social-emotional behavior that is meaningful for later development. The findings help unpack the differential impacts of cardiac vagal tone and cardiac vagal reactivity on dyadic behavior and highlight the importance of considering physiology as a moderator of social-emotional processes.

\section{Appendix}

Coordination of mother-infant dyad during interaction task: indexes extent to which dyad shares the experience of the toy-offer episode, with exchanges that are smooth and mutual behaviors. Coded on a 5-pt Likert scale, over the entire $3 \mathrm{~min}$ of toy-offer episode.

Coordinated, sharing include mom's verbal communication directed to infant or about infant's behavior (ie. "Look, baby"; "What a nice toy"; "You like that toy, huh?"), mom's nonverbal communication including gaze and looks (sharing in joint attention, following baby's gaze or points), facial expressions directed to infant, physical contact with/positioning of infant, and infant's nonverbal communication including gaze and looks (joint attention), vocal signals (both positive and negative calls), gestural signals (pointing, pulling on mom), and postural signals (leaning toward or into mom). 
Important note: The focus is on the dyad and not on either individual. Mothers likely put forth more communication or behaviors, but communication or behavior from mother or infant are equally valid. The heart of the coding is in how the partners share the experience with each other.

\section{Code Behaviors}

4 Maximum coordination

Consistent sharing/coordinated verbal and nonverbal communication between dyad. There is a clear, connected back-and-forth quality as the dyad interacts

3 Strong coordination

Predominant dyadic state is sharing the experience and most communication between the dyad is coordinated and smooth. A few disconnected episodes may still be exhibited.

2 Moderate coordination

Dyad exhibits more coordinated than disjointed episodes.

$1 \quad$ Mild coordination

Dyad exhibits both coordinated and disjointed episodes in roughly equal measure. One partner may make multiple attempts at connection/coordination, but attempts are not consistently reciprocated by the partner.

0 Minimal coordination or does not occur at all.

Dyad makes few if any attempts at sharing or communicating. If present, attempts tend to be disjointed and do not flow smoothly.

\section{References}

Aiken, L., \& West, S. (1991). Multiple regression: Testing and interpreting interactions. Newbury Park: Sage. Ainsworth, M. S. (1979). Infant-mother attachment. American Psychologist, 34, 932-937.

Alkon, A., Lippert, S., Vujan, N., Rodriquez, M. E., Boyce, W. T., \& Eskenazi, B. (2006). The ontogeny of autonomic measures in 6- and 12-month-old infants. Developmental Psychobiology, 48, 197-208. doi:10. 1002/dev.20129.

Bar-Haim, Y., Marshall, P. J., \& Fox, N. A. (2000). Developmental changes in heart period and high-frequency heart period variability from 4 months to 4 years of age. Developmental Psychobiology, 37, 44-56. doi: 10.1002/1098-2302(20007)37:1<44::AID-DEV6.3.0.CO;2-7.

Beauchaine, T. (2001). Vagal tone, development, and Gray's motivational theory: Toward an integrated model of autonomic nervous system functioning in psychopathology, Development and Psychopathology, 183214. Retrieved from http://www.ncbi.nlm.nih.gov/pubmed/11393643

Beauchaine, T. (2015). Respiratory sinus arrhythmia: a transdiagnostic biomarker of emotion dysregulation and psychopathology. Current Opinions in Psychology, 3, 43-48. doi:10.1016/j.copsyc.2017.01.017.

Beauchaine, T., \& Thayer, J. F. (2015). Heart rate variability as a transdiagnostic biomarker of psychopathology. International Journal of Psychophysiology, 98, 338-350. doi:10.1016/j.ijpsycho.2015.08.004.

Belsky, J., \& Pasco Fearon, R. M. (2002). Infant-mother attachment security, contextual risk, and early development: a moderational analysis. Development and Psychopathology, 14, 293-310. doi:10.1017/ s0954579402002067.

Blandon, A. Y., Calkins, S. D., Keane, S. P., \& O’Brien, M. (2008). Individual differences in trajectories of emotion regulation processes: the effects of maternal depressive symptomatology and children's physiological regulation. Developmental Psychology, 44, 1110-1123. doi:10.1037/0012-1649.44.4.1110.

Bohlin, G., Hagekull, B., \& Rydell, A. (2000). Attachment and social functioning: a longitudinal study from infancy to middle childhood. Social Development, 9, 24-39. doi:10.1111/1467-9507.00109.

Boldt, L. J., Kochanska, G., Yoon, J. E., \& Nordling, J. K. (2014). Children's attachment to both parents from toddler age to middle childhood: links to adaptive and maladaptive outcomes. Attachment \& Human Development, 16, 211-229. doi:10.1080/14616734.2014.889181. 
Bowlby, J. (1982). Attachment and loss: Vol. I. Attachment (2nd ed.). New York: Basic Books.

Butler, E., Wilhelm, F. H., \& Gross, J. J. (2006). Respiratory sinus arrhythmia, emotion, and emotion regulation during social interaction. Psychophysiology, 43, 612-622. doi:10.1111/j.1469-8986.2006. 00467.x.

Calkins, S. D., \& Fox, N. A. (1992). The relations among infant temperament, security of attachment, and behavioral inhibition at twenty-four months. Child Development, 63, 1456-1472. doi:10.1111/j.14678624.1992.tb01707.x.

Cipriano, E. A., Skowron, E. A., \& Gatzke-Kopp, L. M. (2011). Preschool children's cardiac reactivity moderates relations between exposure to family violence and emotional adjustment. Child Maltreatment, 16, 205-215. doi:10.1177/1077559511408887.

Cole, P. M., Barrett, K. C., \& Zahn-Waxler, C. (1992). Emotion displays in two-year-olds during mishaps. Child Development, 63, 314-324. doi:10.1111/j.1467-8624.1992.tb01629.x.

Conradt, E., \& Ablow, J. (2010). Infant physiological response to the still-face paradigm: contributions of maternal sensitivity and infants' early regulatory behavior. Infant Behavior and Development, 33, 251265. doi:10.1016/j.infbeh.2010.01.001.

Conradt, E., Measelle, J., \& Ablow, J. C. (2013). Poverty, problem behavior, and promise: differential susceptibility among infants reared in poverty. Psychological Science, 24, 235-242. doi:10.1177/ 0956797612457381.

Deater-Deckard, K., \& Petrill, S. A. (2004). Parent-child dyadic mutuality and child behavior problems: an investigation of gene-environment processes. Journal of Child Psychology and Psychiatry, 45, 11711179. doi:10.1111/j.1469-7610.2004.00309.x.

Demaree, H. A., \& Everhart, D. E. (2004). Healthy high-hostiles: reduced parasympathetic activity and decreased sympathovagal flexibility during negative emotional processing. Personality and Individual Differences, 36, 457-469. doi:10.1016/s0191-8869(03)00109-0.

Dierckx, B., Tulen, J. H. M., Tharner, A., Jaddoe, V. W., Hofman, A., Verhulst, F. C., \& Tiemeier, H. (2011). Low autonomic arousal as vulnerability to externalizing behavior in infants with hostile mothers. Psychiatry Research, 185, 171-175. doi:10.1016/j.psychres.2009.09.014.

Eisenberg, N., Sulik, M. J., Spinrad, T. L., Edwards, A., Eggum, N. D., Liew, J., Sallquist, J., Popp, T. K., Smith, C. L., \& Hart, D. (2012). Differential susceptibility and the early development of aggression: interactive effects of respiratory sinus arrhythmia and environmental quality. Developmental Psychology, 48, 755-768. doi:10.1037/a0026518.

Feldman, R., Greenbaum, C. W., \& Yirmiya, N. (1999). Mother-infant affect synchrony as an antecedent of the emergence of self-control. Developmental Psychology. doi:10.1037/0012-1649.35.1.223.

Ferrer, E., \& Helm, J. L. (2013). Dynamical systems modeling of physiological coregulation in dyadic interactions. International Journal of Psychophysiology, 88, 296-308. doi:10.1016/j.ijpsycho.2012.10. 013.

Frankel, K. A., \& Bates, J. E. (1990). Mother-toddler problem solving: antecedents in attachment, home behavior, and temperament. Child Development, 61, 810-819. doi:10.1111/j.1467-8624.1990.tb02823.x.

Frazier, T. W., Strauss, M. E., \& Steinhauer, S. R. (2004). Respiratory sinus arrhythmia as an index of emotional response in young adults. Psychophysiology, 41, 75-83. doi:10.1046/j.1469-8986. 2003.00131.x.

Friedman, B. H., \& Thayer, J. F. (1998). Autonomic balance revisited: panic anxiety and heart rate variability. Journal of Psychosomatic Research, 44, 133-151. doi:10.1016/s0022-39999(97)00202-x.

Gaziano, P. A., Reavis, R. D., Keane, S. P., \& Calkins, S. D. (2007). The role of emotion regulation and children's early academic success. Journal of School Psychology, 45, 3-19. doi:10.1016/j.jsp.2006.09.002.

Gentzler, A. L., Santucci, A. K., Kovacs, M., \& Fox, N. A. (2009). Respiratory sinus arrhythmia predicts emotion regulation and depressive symptoms in at-risk and control children. Biological Psychology, 82, 156-163. doi:10.1016/j.biopsycho.2009.07.002.

Grossmann, K. E., Grossmann, K., \& Zimmermann, P. (1999). A wider view of attachment and exploration: Stability and change during the years of immaturity. In J. Cassidy \& P. R. Shaver (Eds.), Handbook of attachment: Theory, research, and clinical applications (pp. 760-786). New York: Guilford Press.

Ham, J., \& Tronick, E. (2006). Infant resilience to the stress of the still-face. Annals of the New York Academy of Sciences, 1094, 297-302. doi:10.1196/annals.1376.038.

Hastings, P. D., Sullivan, C., McShane, K. E., Coplan, R. J., Utendale, W. T., \& Wyncke, J. D. (2008). Parental socialization, vagal regulation, and preschoolers' anxious difficulties: direct mothers and moderated fathers. Child Development, 79, 45-64. doi:10.1111/j.1467-8624.2007.01110.x. 
Holochwost, S. J., Gariepy, J.-L., Propper, C. B., Mills-Koonce, W. R., \& Moore, G. A. (2014). Parenting behaviors and vagal tone at six months predict attachment disorganization at twelve months. Developmental Psychobiology, 56, 1423-1430. doi:10.1002/dev.21221.

Houtveen, J. H., Rietveld, S., \& de Geus, E. J. C. (2002). Contribution of tonic vagal modulation of heart rate, central respiratory drive, respiratory depth, and respiratory frequency to respiratory sinus arrhythmia during mental stress and physical exercise. Psychophysiology, 39, 427-436. doi:10.1111/1469-8986. 3940427.

Huffman, L. C., Bryan, Y. E., del Carmen, R., Pedersen, F. A., Doussard-Roosevelt, J. A., \& Porges, S. W. (1998). Infant temperament and cardiac vagal tone: assessments at twelve weeks of age. Child Development, 69, 624-635. doi:10.1111/j.1467-8624.1998.tb06233.x.

Kochanska, G., Forman, D. R., \& Coy, K. C. (1999). Implications of the mother-child relationship in infancy for socialization in the second year of life. Infant Behavior and Development, 22, 249-265. doi:10.1016/ s0163-6383(99)00009-0.

Laible, D. J., \& Thompson, R. A. (1998). Attachment and emotional understating in preschool children. Developmental Psychology, 34, 1038-1045. doi:10.1037/0012-1649.34.5.1038.

Licht, C. M. M., de Geus, E. J. C., Zitman, F. G., Hoogendijk, W. J. G., van Dyck, R., \& Penninx, B. W. J. H. (2008). Association between major depressive disorder and heart rate variability in the Netherlands study of depression and anxiety (NESDA). Archives of General Psychiatry, 65, 1358-1367. doi:10.1001/ archpsyc.65.12.1358.

Lindsey, E. W., Mize, J., \& Pettit, G. S. (1997). Mutuality in parent-child play: consequences for children's peer competence. Journal of Social and Personal Relationships, 14, 523-538. doi:10.1177/ 0265407597144007.

Lindsey, E. W., Cremeens, P. R., Colwell, M. J., \& Caldera, Y. M. (2009). The structure of parent-child dyadic synchrony in toddlerhood and children's communication competence and self-control. Social Development, 18, 375-396. doi:10.1111/j.1467-9507.2008.00489.x.

Oosterman, M., de Schipper, J. C., Fisher, P., Dozier, M., \& Schuengel, C. (2010). Autonomic reactivity in relation to attachment and early adversity among foster children. Development and Psychopathology, 22, 109-118. doi:10.1017/s0954579409990290.

Oveis, C., Cohen, A. B., Gruber, J., Shiota, M. N., Haidt, J., \& Keltner, D. (2009). Resting respiratory sinus arrhythmia is associated with tonic positive emotionality. Emotion, 9, 265-270. doi:10.1037/a0015383.

Porges, S. W. (1995). Orienting in a defensive word: mammalian modifications of our evolutionary heritage: a polyvagal theory. Psychophysiology, 32, 301-318. doi:10.1111/j.1469-8986.1995.tb01213.x.

Porges, S. W. (2007). The polyvagal perspective. Biological Psychology, 74, 116-142. doi:10.1016/j. biopsycho.2006.06.009.

Richards, J. E. (1987). Infant visual sustained attention and respiratory sinus arrhythmia. Child Development, 58, 488-496. Retrieved from http://www.jstor.org/stable/1130525.

Spinrad, T. L., Eisenberg, N., Cumberland, A., Fabes, R. A., Valiente, C., Shepard, S. A., Reiser, M., Losoya, S. H., \& Guthre, I. L. K. (2006). Relation to emotion-related regulation to children's social competence: a longitudinal study. Emotion, 6, 498-510. doi:10.1037/1528-3542.6.3.498.

Stifter, C. A., Fox, N. A., \& Porges, S. W. (1989). Facial expressivity and vagal tone in 5- and 10-month-old infants. Infant Behavior and Development, 12, 127-137. doi:10.1016/0163-6383(89)90001-5.

Teti, D. M., \& McGourty, S. (1996). Mothers versus trained observers in assessing children's secure base behavior: theoretical and methodological considerations. Child Development, 67, 597-605. doi:10.1111/j. 1467-8624.1996.tb01753.x.

Thompson, R. A. (1994). Emotion regulation: a theme in search of definition. Monographs of the Society for Research in Child Development, 59, 25-52. doi:10.1111/j.1540-5834.1994.tb01276.x.

Waters, E., \& Deane, K. E. (1985). Defining and assessing individual differences in attachment relationships: Q-methodology and the organization of behavior in infancy and early childhood. Monographs of the Society for Research in Child Development, 50, 41-65. doi:10.2307/3333826.

Waters, S. F., West, T. V., \& Mendes, W. B. (2014). Stress contagion: physiological covariation between mothers and infants. Psychological Science, 25, 934-942. doi:10.1177/0956797613518352. 